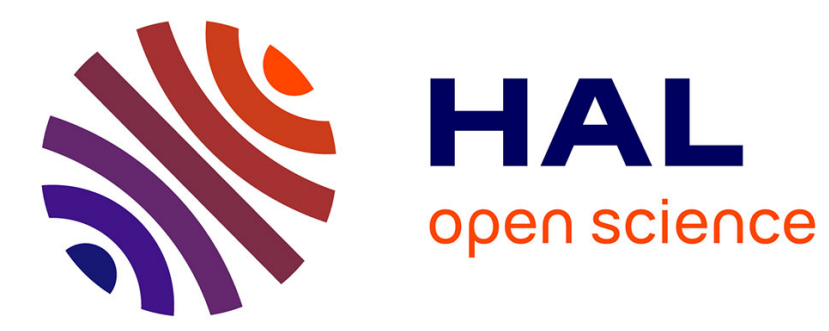

\title{
Ordering Frustration in Large-Scale Co-Pt Nanoalloys
}

Alexis Front, Christine Mottet

\section{To cite this version:}

Alexis Front, Christine Mottet. Ordering Frustration in Large-Scale Co-Pt Nanoalloys. Journal of Physical Chemistry C, 2021, 125 (29), pp.16358 - 16365. 10.1021/acs.jpcc.1c05701 . hal-03307140

\section{HAL Id: hal-03307140 https://hal.science/hal-03307140}

Submitted on 13 Oct 2021

HAL is a multi-disciplinary open access archive for the deposit and dissemination of scientific research documents, whether they are published or not. The documents may come from teaching and research institutions in France or abroad, or from public or private research centers.
L'archive ouverte pluridisciplinaire HAL, est destinée au dépôt et à la diffusion de documents scientifiques de niveau recherche, publiés ou non, émanant des établissements d'enseignement et de recherche français ou étrangers, des laboratoires publics ou privés. 


\title{
Ordering Frustration in Large-Scale Co-Pt Nanoalloys
}

\author{
Alexis Front and Christine Mottet*
}

Cite This: J. Phys. Chem. C 2021, 125, 16358-16365

Read Online

ACCESS 1

Wlll Metrics \& More

回 Article Recommendations

ABSTRACT: Large-scale nanoalloys of Co-Pt from 3 to $7 \mathrm{~nm}$ are modeled using realistic atomistic potentials and Monte Carlo simulations, sweeping the entire concentration range. They display a clear tendency to reproduce bulk-ordered phases in their core- and surface-ordered superstructures on their facets. Most of the ordered facets are simply the termination of the core-ordered phase but one of them, namely, $(\sqrt{3}$ $\times \sqrt{3}) \mathrm{R} 30^{\circ}(111)$, is not compatible with the core structure, inducing disorder underneath (111) facets. At equiconcentration, the $\mathrm{L}_{0}$ phase, within the truncated octahedral shape, displays (100) facets leading to both pure and mixed variants with no surface rearrangements. However, we predict mixed composition on all (100) facets, as for the infinite (100) surfaces, indicating a chemical ordering frustration in nanoalloys. For 5fold symmetric structures like decahedra and icosahedra, the core ordering remains inside the multiply twinned tetrahedra forming the decahedral or icosahedral structure.

\section{INTRODUCTION}

Nanoalloys ${ }^{1}$ combine finite size effects and alloy effects, significantly extending the range of nanomaterial properties. ${ }^{2-4}$ A crucial step for the control of these properties is to characterize structural and chemical arrangements and link these with functional and mechanical properties. Experiments have made impressive progress to synthesize ${ }^{5}$ and characterize nanoalloys of controlled size, morphology, and composition. It is now possible to determine by aberration-corrected highresolution microscopy techniques, the nature of the segregated element at the surface of the cluster. ${ }^{6-8}$ However, the precise chemical arrangement at the surface is still challenging to observe, meaning experiments, such as in situ grazing incidence $\mathrm{X}$-ray diffusion ${ }^{9,10}$ or scanning transmission electron microscopy $^{7,8}$ with high-angle annular dark-field imaging, often need simulations to refine their interpretations. Co-Pt nanoalloys have been extensively studied for more than 15 years because of their potential applications in catalysis and ultrahigh density magnetic storage, giving rise to a review devoted to that subject. ${ }^{11}$ In fuel-cell applications, Co-Pt nanoalloys present two advantages. The first is to reduce the amount of $\mathrm{Pt}$ and, therefore, reduce the cost, which is essential for the commercialization of zero-emission vehicles. ${ }^{12}$ The second is that it presents higher activity in the electrochemical oxygen reduction reaction as compared to pure Pt. In particular, the $\mathrm{L}_{0}$ ordered phase of the CoPt alloys has been measured to be seven times more active than the disordered phase. ${ }^{13}$ Chemical ordering of the bimetallic nanoparticles is also crucial in magnetic applications to maintain the magnetic moment orientation of one nanoparticle corresponding to one macro- spin. ${ }^{14}$ Indeed, the $\mathrm{L1}_{0}$ ordered phase constituted by an alternance of pure $\mathrm{Pt} /$ pure Co atomic layers sensibly increases the magnetic anisotropy in bulk alloys, which is also expected for nanoalloys. ${ }^{15-17}$ There is, thus, detailed knowledge of how precise chemical ordering in the core and on the surface of the bimetallic nanoparticles is crucial to control their properties.

However, it was not evident that small nanoparticles (of diameter $2-3 \mathrm{~nm}$ ) can be ordered with the $\mathrm{L1}_{0}$ phase like the bulk alloy. ${ }^{18}$ Indeed, it has first been shown experimentally that nanoparticles had to be heated during or after their synthesis to reach the equilibrium phase, which was indeed $L 1_{0}$ in the facecentered cubic (fcc) structure. ${ }^{9,10,19-27}$ This does not exclude the presence of other structures such as decahedra or icosahedra at small sizes, but even in the case of decahedra, some locally nano-L $1_{0}$ phases have been observed. ${ }^{26}$ Theoretical studies ${ }^{28-33}$ have been undertaken, sometimes in collaboration with experiments, ${ }^{10,22}$ to better understand and identify the chemical ordering, with possible competition from surface segregation, which experimental observations could not always characterize. More recently, using X-ray photoelectron spectroscopy and a theoretical model based on electronic structure calculations, it has been possible to identify Pt surface segregation with specific surface site preferences and Co

Received: June 28, 2021

Published: July 16, 2021 
subsurface segregation, ${ }^{34}$ without the occurrence of the $\mathrm{L1}_{0}$ ordered phase in the core, which is in contradiction with previous studies. As a result, important uncertainties remain concerning the chemical ordering and surface segregation effects in Co-Pt nanoalloys. We propose, here, a new theoretical study, based on a realistic interatomic potential ${ }^{35}$ able to reproduce all of the ordered phases of the bulk phase diagram and the surface segregation of the semi-infinite alloyed surfaces. Using Monte Carlo simulations in the appropriate thermodynamical ensemble, we present a study on the whole range of concentration for large simulated system sizes of 3-7 $\mathrm{nm}$ and different morphologies, which have never been performed up to now.

We predict the presence of chemical ordered configurations with a truncated octahedral (TOh) morphology with a facecentered cubic structure, a decahedral (Dh) structure composed of twinned tetrahedra sharing a common 5-fold symmetry axis, and icosahedral (Ih) structures made of 20 twinned tetrahedra, all pointing toward the center of the cluster.

These three motifs are the most competitive, usually stabilized with $\mathrm{Ih}$ at small sizes, $\mathrm{Dh}$ at intermediate sizes, and finally TOh at large sizes. ${ }^{36}$ As the energy difference between one motif to the other is very small, a finite system and sample size fluctuations can prevent the precise characterization of size transitions, with all motifs observed in a range of sizes between 3 and $5 \mathrm{~nm} .{ }^{26}$ In the following, we just illustrate chemical ordering effects for all of these most probable shapes. We performed semigrand canonical Monte Carlo simulations with a freely variable concentration, which is equilibrated within the Monte Carlo algorithm to find the proper chemical phase at equilibrium with fixed mixed chemical potential.

To analyze finite size effects, we developed a multisite approach where we compare the cluster core chemical ordering to the bulk phases of $\mathrm{Co}-\mathrm{Pt}$ alloys and possible surface segregation and/or reconstruction of the cluster facets to the equivalent alloy surfaces.

\section{THEORETICAL MODEL AND NUMERICAL SIMULATIONS}

To model the Co-Pt nanoalloys, we use a semiempirical potential derived from the tight-binding framework within the approximation of the density-of-states to its second moment. ${ }^{37}$ Such potentials have been tested for alloy surfaces and nanoalloys, in general, for systems with ordering tendency ${ }^{38}$ as for systems with demixion tendency. ${ }^{39,40}$ Here, we use a parametrization for the $\mathrm{Co}-\mathrm{Pt}$ system, ${ }^{35}$ which is able to reproduce a bulk phase diagram and surface segregation, notably the segregation reversal between (111) and (110) surfaces. ${ }^{41,42}$ These are important conditions for the interatomic potential to predict chemical configurations of nanoalloys. The equilibrium configurations of $\mathrm{Co}_{1-c} \mathrm{Pt}_{c}$ nanoparticles are determined by Monte Carlo simulations in the semigrand canonical ensemble, where $c$ is the Pt concentration.

Many-Body Potential for the Co-Pt System. The energetic model is a semiempirical many-body potential derived from the density-of-states of the d-band metals (transition metals) approximated to its second moment (width of the d-band) in the tight-binding framework. ${ }^{37}$ It leads to an attractive term with a square root dependence on the coordination number

$$
E_{i}^{\text {band }}=-\sqrt{\sum_{j, r_{i j}<r_{a b}^{c}} \xi_{a b}^{2} \mathrm{e}^{-2 q_{a b}\left(\frac{r_{i j}}{r_{a b}^{0}}-1\right)}}
$$

for the band term at site $i$, where $a, b=(A, B)$ is the nature of the metal atom, $r_{i j}$ is the distance between the atom at site $i$ and one neighbor at site $j, r_{a b}^{c}$ is the cutoff distance, and $r_{a b}^{0}$ is the first-neighbor distance depending on the nature of the atoms. A repulsive term of the Born-Mayer type is added to simulate the core repulsion

$$
E_{i}^{\text {rep }}=\sum_{j, r_{i j}<r_{a b}^{c}} A_{a b} \mathrm{e}^{-p_{a b}\left(\frac{r_{i j}}{r_{a b}^{0}}-1\right)}
$$

The homoatomic parameters $p_{a b}, q_{a b}, A_{a b}, \xi_{a b}$ with $a=b$ are fitted to the cohesive energies, lattice parameters, and elastic constants. The heteroatomic parameters $(a \neq b)$ are fitted to the mixing energies calculated using the density functional theory and to experimental data such as the order/disorder critical temperatures ${ }^{43,44}$ of the bulk alloys and the surface segregation of alloy surfaces. The details are given in ref 35 and the parameters are reported in Table 1.

Table 1. Parameters of the Tight-Binding Many-Body Potential

\begin{tabular}{ccccc}
$a-b$ & $p_{a b}$ & $q_{a b}$ & $A_{a b}$ & $\xi_{a b}$ \\
$\mathrm{Co}-\mathrm{Co}$ & 8.6420 & 2.3000 & 0.1582 & 1.8443 \\
$\mathrm{Pt}-\mathrm{Pt}$ & 10.7960 & 3.1976 & 0.1993 & 2.2318 \\
$\mathrm{Co}-\mathrm{Pt}$ & 9.7190 & 2.7488 & 0.2750 & 2.4880 \\
\hline
\end{tabular}

The Co-Pt bulk phase diagram presents three ordered phases around the compositions $\mathrm{Co}_{3} \mathrm{Pt}$, CoPt, and $\mathrm{CoPt}_{3}$, with lower stability of the Co-rich phase than the Pt-rich one. The surfaces present chemical-ordered superstructures, some of them being the termination of bulk-ordered phases, while others being original and exclusively suited to the surface. As in the experimental observations (see ref 35 ), the Co-Pt alloy surfaces display Pt surface segregation on the (111) and (100) orientations and Co surface segregation on the (110) surface.

Monte Carlo Simulations. Monte Carlo simulations are performed in the semigrand canonical ensemble using the Metropolis algorithm, which means that the total number of atoms in the binary system, the temperature, and the pressure are fixed, whereas the number of atoms per species can change. As a consequence, the concentration at equilibrium is a result of the Monte Carlo process. In the Metropolis algorithm, starting with the system in the " $i$ " state, with energy $E(i)$, one performs one atomic trial, which can be either moving an atom or permuting the chemical nature of an atom, we calculate the new energy $E(j)$. If $E(j)$ is lower than $E(i)$, the new configuration is accepted and the simulation goes on starting from it, else, the " $j$ " state is accepted according to the following probability

$$
\exp \left[-\frac{E(j)-E(i)+\mathrm{d} n\left(\mu_{B}-\mu_{A}\right)+\frac{3}{2} \ln \left(\frac{m_{B}}{m_{A}}\right)}{k_{\mathrm{B}} T}\right]
$$

where $\mathrm{d} n$ is the permutation sign equal to 1 if we permute an atom of type $A$ into an atom of type $B$ or -1 if we permute an atom of type $B$ into an atom of type $A . m_{A}$ and $m_{B}$ are the atomic masses of the elements $A$ and $B$. 
In a standard Monte Carlo simulation with $N$ atoms, we run 5-10000 macrosteps after leaving the system being equilibrated during 2-5000 steps. One macrostep consists of randomly proposing either displacements of atoms or permutations of chemical species, $\mathrm{N}$-times. We usually start with a Co-pure or Pt-pure system and increase or decrease the chemical potential difference $\mu_{B}-\mu_{A}$ to scan all of the concentration ranges, and we start the new Monte Carlo run for each chemical potential from the last configuration.

\section{RESULTS AND DISCUSSION}

Truncated Octahedra (TOh). We performed Monte Carlo simulations in the whole concentration range for $\mathrm{Co}-\mathrm{Pt}$ clusters of three different sizes: 1289 atoms (3.3 nm), 2951 atoms $(4.4 \mathrm{~nm})$, and 9879 atoms $(6.8 \mathrm{~nm})$, as illustrated in Figure 1. First, we investigated chemical ordering in the core of

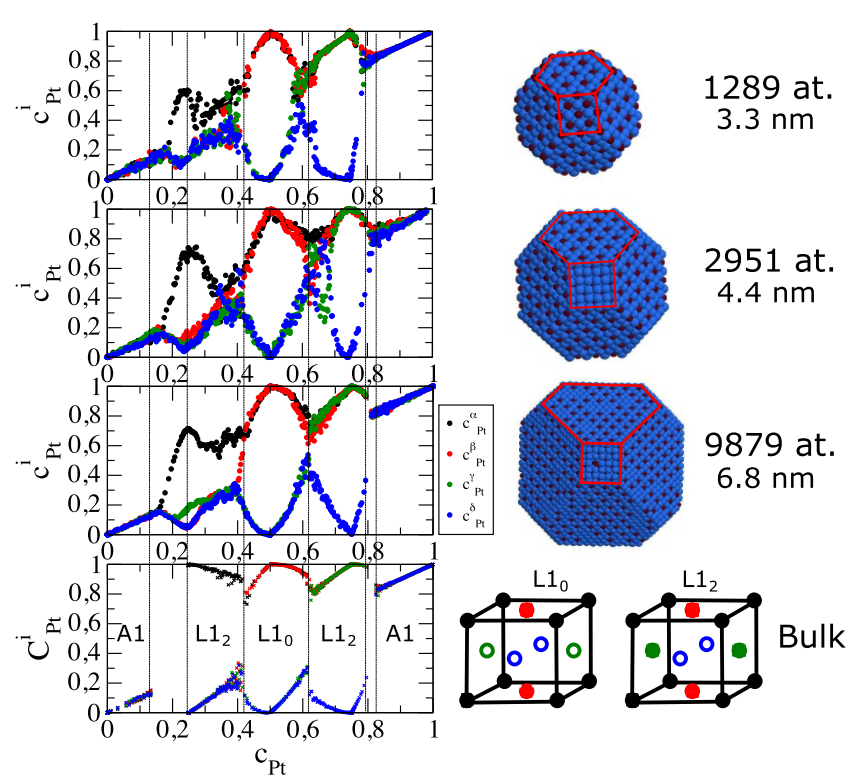

Figure 1. Core sublattice Pt concentration $c_{\mathrm{Pt}}^{i}$ (with $i$ nominating the four sublattices $\alpha, \beta, \gamma$, and $\delta$ ) of truncated octahedra (TOh) of 1289 atoms $(3.3 \mathrm{~nm}), 2951$ atoms $(4.4 \mathrm{~nm})$, and 9879 atoms $(6.8 \mathrm{~nm})$ as compared to the bulk ones (bottom graph) at $300 \mathrm{~K} . \mathrm{L}_{0}$ and $\mathrm{Ll}_{2}$ ordered phases are characterized by their lattice mesh with four colors corresponding to the four sublattices of the fcc structure. In the lattice mesh, full circles correspond to sites occupied by one species and empty ones by the other species.

the nanoalloys as compared to the bulk-ordered phase. The $\mathrm{Co}-\mathrm{Pt}$ bulk phase diagram displays three ordered phases with the $\mathrm{L1}_{0}$ type at $50 \%$, an alternance of pure atomic layers in the (001) direction, and the $\mathrm{L}_{2}$ type at $25 / 75 \%$, an alternance of pure and mixed atomic layers in the same direction (see the lattice mesh in Figure 1). To characterize these chemical ordered phases, we plotted the occupation of the four fcc sublattices in the bulk and in the clusters. Starting with the bulk (bottom graph in Figure 1), we notice well-separated domains depending on the concentration, and some miscibility gaps where there is no stable phase but a coexistence of the two adjacent phases. At a low Pt concentration, the four sublattices are equally occupied, this means that the phase is a disordered Co-rich solid solution. Then, by increasing the $\mathrm{Pt}$ concentration, we see that one over four is fully occupied by $\mathrm{Pt}$, the other three by $\mathrm{Co}$; this corresponds to the $\mathrm{L}_{2}$ phase at $25 \% \mathrm{Pt}$ corresponding to the $\mathrm{Co}_{3} \mathrm{Pt}$ compounds. The domain of the existence of this phase is extended up to around $40 \% \mathrm{Pt}$. Then, between 40 and $60 \% \mathrm{Pt}$, two over the four sublattices are occupied by $\mathrm{Pt}$, the other two by Co, corresponding to the $\mathrm{L} 1_{0}$ phase at equiconcentration. Then, going further in the $\mathrm{Pt}$ concentration, we observe the same $\mathrm{L}_{2}$ phase but Pt-rich corresponding to the $\mathrm{CoPt}_{3}$ compounds, which is stable in a domain between 60 and $80 \%$ Pt. Finally, the four sublattices are equally occupied, corresponding to the Pt-rich solid solution. Now, what does happen in the core of the nanoparticles? We notice in Figure 1 that sublattice occupations in the core of the different clusters follow quite well the ones of the bulk; for example, we clearly see $\mathrm{L}_{0} \mathrm{CoPt}$ around equiconcentration and $\mathrm{Ll}_{2} \mathrm{CoPt}_{3}$ in the Pt-rich side, but this is less comparable in the Co-rich side. In that case, the large miscibility gap in the bulk is not reproduced in the clusters. Even by increasing the size from 3 to $7 \mathrm{~nm}$, the $\mathrm{L}_{2}$ phase becomes more clearly ordered but there is still no miscibility gap. This is a clear sign of the finite size effect where the phase coexistence is not allowed as in the infinite bulk system. The loss of chemical ordering observed on nanoalloys as compared to bulk alloys results from surface reconstruction and, therefore, chemical frustration in the core at the vicinity of the surface, which will be discussed in detail in the following.

A central challenge when passing from periodic lattice supercells to finite size clusters is that the cluster shape can influence the overall structure. It can change the structure from an fcc octahedron to 5-fold symmetry structures like an icosahedron or a decahedron but it can also influence the chemical ordering even if the bulk symmetry is conserved like on the fcc octahedron. In such cases, the system has to accommodate the finite size with a given morphology, notably the coexistence of (100) and (111) facets, and eventual surface superstructures, which do not exist in bulk. Not only the cluster surface can be affected but because of the surface, the chemical ordering in the core is also affected. So the explanation of cluster core disordering can have its origin from the surface shell of the nanoparticles. We will consider now the different facets of the TOh clusters.

From the outputs of the semigrand canonical Monte Carlo simulations performed at $300 \mathrm{~K}$, we extract the (100) facets concentration in $\mathrm{Pt}$ for the two cluster sizes and compare them to the surface concentration of the (100) infinite surface. ${ }^{35}$ In Figure 2, we see that the (100) infinite surface displays a plateau over a large concentration range from $25 \% \mathrm{Pt}$ to $75 \%$ $\mathrm{Pt}$, which corresponds to the $\mathrm{c}(2 \times 2)$ surface reconstruction ${ }^{35}$ (see the scheme in Figure 2). In this surface-ordered structure, each square sublattice is occupied by the same type of atom. We plotted the sublattice occupations for the two cluster sizes in Figure 2, and we noticed that this $\mathrm{c}(2 \times 2)$ structure is also well characterized on the clusters, on approximately the same concentration range as the infinite surface. So, we can conclude that $\mathrm{c}(2 \times 2)$ appears on clusters with variable sizes, as we can notice on the snapshots of $\mathrm{TOh}_{1289}$ in Figure 2. The agreement between the concentration on cluster facets compared to the infinite surface is almost perfect for the larger size $\left(\mathrm{TOh}_{2951}\right)$. In the case of the smaller cluster, the (100) facet concentration has two plateaus, whereas on increasing cluster size, the facet behavior tends to the surface behavior. It is worth noticing that the (100) facet of $\mathrm{TOh}_{1289}$ is a square of $3 \times 3$ atoms, whereas the (100) facet of $\mathrm{TOh}_{2951}$ has $4 \times 4$ atoms (see Figure 1 ). In the case of facets with an odd number of atoms (as for the $\left.\mathrm{TOh}_{1289}\right)$, the number of $\mathrm{Co}$ and $\mathrm{Pt}$ atoms can not be equal, resulting in a transition at $35 \% \mathrm{Pt}$ between $\mathrm{c}(2 \times 2) \mathrm{Pt}$-rich (a) 

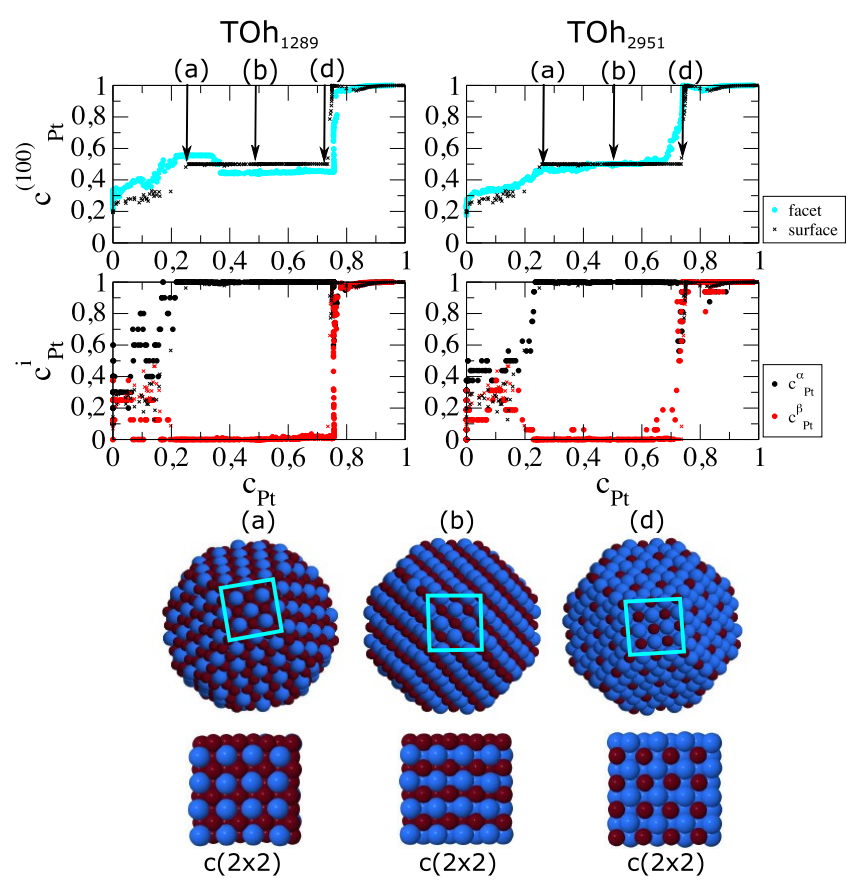

Figure 2. (100) facet Pt concentration of $\mathrm{TOh}_{1289}$ (left) and $\mathrm{TOh}_{2951}$ (right) at $300 \mathrm{~K}$. The graphs in the top row represent the global Pt concentration on the (100) facets (blue dots) and on the (100) infinite surface (black crosses) as a function of the core $\mathrm{Pt}$ concentration $c_{\mathrm{Pt}}$. The graphs in the bottom row represent the sublattice Pt concentration of the (100) facets where two sublattices $(\alpha$ and $\beta$ ) are enough to characterize chemical surface ordering. All along the plateau of the $c_{(100)}$ concentration, each sublattice is fully occupied by one type of atom; the corresponding superstructure is $c(2 \times 2)$, which is illustrated by snapshots of the infinite (100) surface of the different ordered alloys $\mathrm{Co}_{3} \mathrm{Pt}, \mathrm{CoPt}$, and $\mathrm{CoPt}_{3}$ and snapshots for $\mathrm{TOh}_{1289}$ with (a), (b), and (d) corresponding to specific concentrations. The blue square points to the (100) facet. Co atoms are in red and $\mathrm{Pt}$ atoms in blue.

and a $c(2 \times 2)$ Co-rich (b). At the end of the plateau, $c_{\mathrm{Pt}}$ increases continuously to obtain (100) pure Pt facets.

To conclude, the $(100)$ facets present the $c(2 \times 2)$ superstructure, which is a simple prolongation of one (100) mixed layer of the $\mathrm{L}_{2}$ bulk-ordered phase. So, in that case, there is no surface segregation or the reconstruction effect as compared to core ordering. However, at the equiconcentration, $c(2 \times 2)$ on each facet is not compatible with the $\mathrm{L} 1_{0}$ core ordering because in the direction of the alternance of the pure atomic layers, the facets should be pure. This represents a chemical frustration of the core ordering induced by the facet reconstruction. This means that all of the (100) facets are mixed, which can have important consequences for catalytic properties.

The (111) facets present a lot of superstructures, which are illustrated in Figure $3 a-d . p(2 \times 1)$ at equiconcentration (Figure $3 \mathrm{~b}$ ) and $\mathrm{p}(2 \times 2)$ at $75 \% \mathrm{Pt}$ (Figure $3 \mathrm{~d}$ ) are well characterized by the four surface sublattices. At equiconcentration, the four sublattices are occupied two by two, leading to $c(2 \times 1)$, which corresponds to a typical (111) layer of the bulk-ordered $\mathrm{L}_{0}$ phase. At $75 \% \mathrm{Pt}$, three of them are occupied by $\mathrm{Pt}$, the other one by $\mathrm{Co}$, leading to $\mathrm{p}(2 \times 2)$, corresponding to a typical (111) layer of the $\mathrm{L} 1_{2}$ bulk-ordered phase. For these concentrations, the cores of the clusters are well ordered, as shown in Figure 1 around 50 and $75 \% \mathrm{Pt}$. There is no ordering frustration by the facets since they adopt exactly the
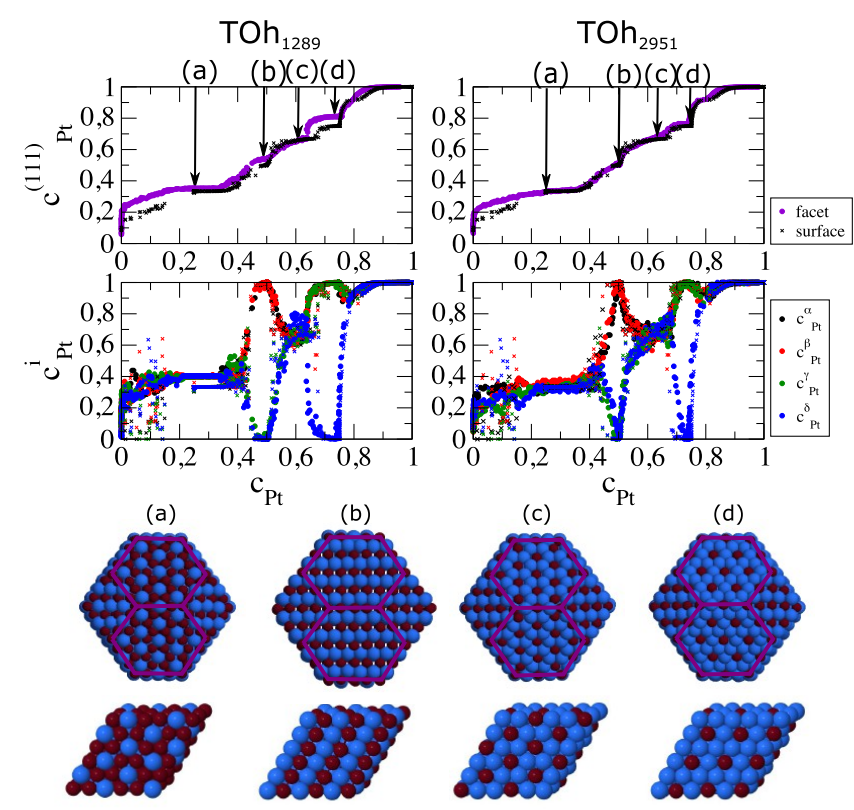

$\mathrm{Pt}_{\mathrm{Pt}}$

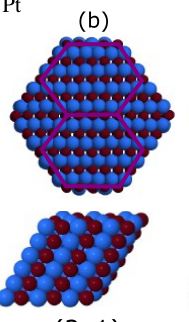

$(\sqrt{ } 3 \times \sqrt{ } 3) R 30$

$\mathrm{p}(2 \times 1)$
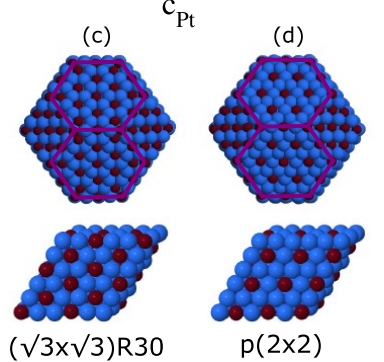

Figure 3. (111) facet Pt concentration of $\mathrm{TOh}_{1289}$ (left) and $\mathrm{TOh}_{2951}$ (right) as compared to the one of the infinite (111) surface of the $\mathrm{Co}-\mathrm{Pt}$ alloy (in black) at $300 \mathrm{~K}$. Same graphs as Figure 2 except there are four sublattices on the (111) surface. Structures $(a-d)$ represent $\mathrm{TOh}_{1289}$ for different concentrations and their corresponding motifs for the (111) facet. (a), (b), and (d) correspond to the same composition, as shown in Figure 2.

same ordering as any atomic plane in the same orientation, which leads to a perfectly ordered cluster in the $\mathrm{CoPt}_{3}$ composition. At equiconcentration, the cluster is almost perfectly ordered as if we extract $\mathrm{TOh}$ from the $\mathrm{L}_{0}$ bulk phase. The only deviation from this perfect ordering takes place on the (100) facets, which are all mixed up to $75 \% \mathrm{Pt}$ and become all pure just above $75 \% \mathrm{Pt}$. Above $80 \% \mathrm{Pt}$, the clusters are disordered on the surface and in the core. At around 30 and $60 \% \mathrm{Pt}$, the four surface sublattices are equally occupied but display a plateau. This does not mean that the (111) facets are disordered but there is another ordering with a larger mesh that can not be characterized by only four sublattices. Indeed, we have seen in a previous study on the infinite $\mathrm{Co}-\mathrm{Pt}$ surfaces ${ }^{35}$ that the (111) infinite surface presents the $(\sqrt{ } 3 \times \sqrt{3}) \mathrm{R} 30^{\circ}$ superstructure, which can be characterized by nine sublattices. We did not determine the occupation of the surface sublattices for the clusters but we see easily in Figure 3a,c that the (111) facets adopt the same superstructure as the $(\sqrt{3} \times \sqrt{3}) \mathrm{R} 30^{\circ}$ on the infinite surface. However, this superstructure does not fit with the chemical ordering of the $\mathrm{L}_{2}$ phase in the core. This leads to a core disordering at least at the vicinity of the (111) facets, but the result is a dispersion in the core sublattice occupations in the $\mathrm{L}_{2}$ domain for the 1289 atom cluster (see Figure 1). When the size increases from 1289 to 2951 atoms, the core is sufficiently large to be relatively ordered and the subsurface contribution is less important. This behavior is exactly a chemical ordering frustration in the cluster because of the finite size effect.

To our knowledge, there is one experimental observation of Pt surface segregation on $\mathrm{CoPt}_{3}$ nanoparticles by Chi et al. ${ }^{7}$ in Vojislav R. Stamenkovic's group where they observed a surface 
platinum-skin-layer formation on $\mathrm{CoPt}_{3}$ nanoparticles of about $8 \mathrm{~nm}$ during in situ annealing using a scanning transmission electron microscope at $620 \mathrm{~K}$, but they can not estimate precisely the skin-layer concentration in Pt. It seems that our prediction of a certain Pt surface segregation could be fully in agreement with their observation, even if in our model, at $75 \%$ $\mathrm{Pt}$ in the core, the cluster surface is not pure in Pt since the (111) facets display the $\mathrm{p}(2 \times 2)$ surface structure.

Decahedra (Dh). In the decahedral (Dh) structure, the nanoparticles lose their translation symmetry as compared to TOh. They display one 5-fold symmetry axis, which can be seen in Figure 4. The Dh morphology displays (111) facets (a)
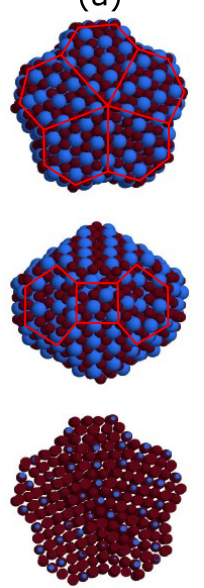

(b)
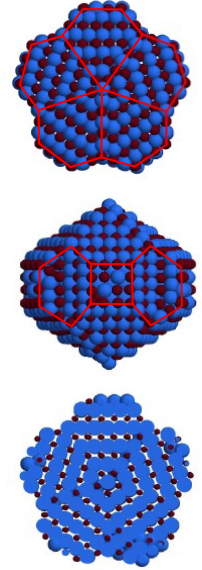

(c)
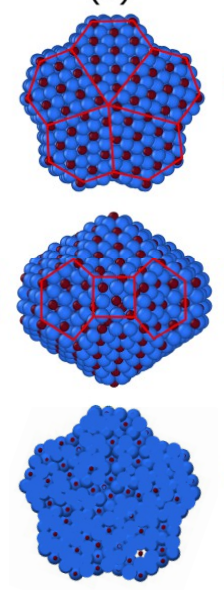

(d)
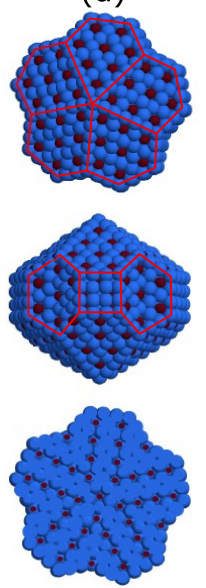

Figure 4. Snapshots of ordered structures $(\mathrm{a}-\mathrm{d})$ of $\mathrm{Dh}_{1308}$ at $300 \mathrm{~K}$, as reported in Figures 5 and 6 . The first and second rows represent shell views, and the third row shows cutting views of the first line with the 5-fold symmetry axis perpendicular to the figure. The red square and the red hexagon point, respectively, to the (100) facet and the (111) facet. Co atoms are in red and Pt atoms in blue.

and truncations parallel to the 5-fold axis, leading to (100) facets and narrow (110) facets to avoid angular edges. In the following, we will essentially consider the (111) facets and the core of the $\mathrm{Dh}$ nanoparticles of 1-3000 atoms.

As before, we compared the chemical ordering in the core and on (111) facets of the decahedra of 1309 and 2802 atoms, respectively, to those of the bulk and the (111) surface of CoPt alloys. The results are illustrated in Figure 5 for the core and in Figure 6 for the (111) facets. As for the TOh structure, the core sublattices in Figure 5 of the Dh clusters reproduce quite well the different ordered phases of the bulk alloy. This demonstrates that although the atomic structure is no longer periodic, instead composed of five tetrahedra sharing one edge along the 5-fold axis, the chemical ordering follows the same as in the infinite alloy. This $\mathrm{L}_{0}$ ordering in each tetrahedron has been observed on FePt and $\mathrm{CoPt}$ nanoparticles with the decahedral symmetry by Tournus et al. ${ }^{26}$ by high-resolution transmission electron microscopy, in very good agreement with the present model.

In the same fashion, the Pt concentration on (111) facets can be compared to the (111) surface concentration of semiinfinite alloys, as shown in Figure 6. We see an almost perfect overlap of the two curves, except on the Co-rich side where $\mathrm{Pt}$ segregation is slightly stronger on (111) facets, but the

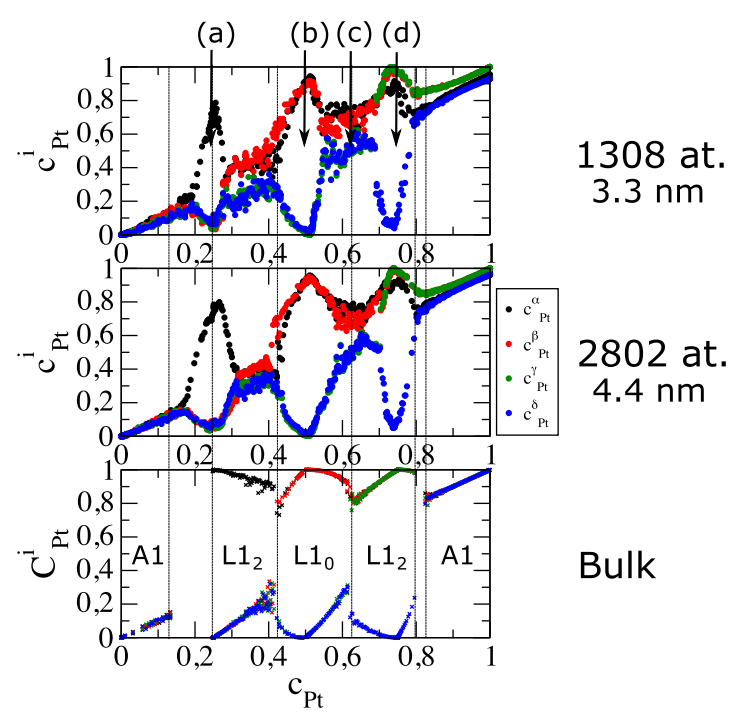

Figure 5. Core sublattice $\mathrm{Pt}$ concentration of $\mathrm{Dh}_{1308}$ (top), $\mathrm{Dh}_{2802}$ (middle), and the bulk (bottom) at $300 \mathrm{~K}$. The structures corresponding to the compositions pointed by $(\mathrm{a}-\mathrm{d})$ are represented in Figure 4 for $\mathrm{Dh}_{1308}$.

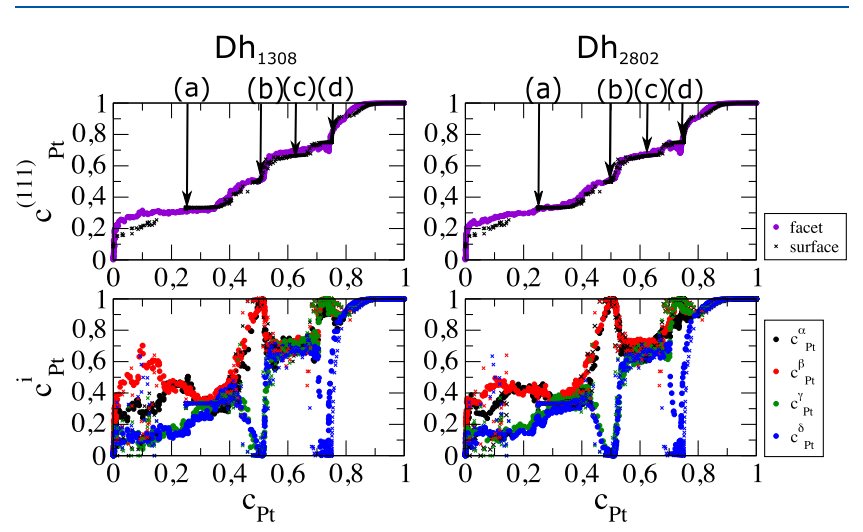

Figure 6. (111) facet Pt concentration of $\mathrm{Dh}_{1308}$ (left) and of $\mathrm{Dh}_{2802}$ (right) as compared to the one of the infinite (111) surface of the Co-Pt alloy (in black) at $300 \mathrm{~K}$. Same graphs as Figure 3. Structures $(\mathrm{a}-\mathrm{d})$ are represented in Figure 4.

difference decreases as the cluster size increases. Here, again, the $\mathrm{Dh}$ clusters display the same chemical ordering on their (111) facets as one of the (111) infinite Co-Pt alloy surfaces and also the same as one of the TOh clusters. The (111) facets of the $\mathrm{Dh}$ can be seen in Figure 4 where each snapshot corresponds to a plateau of Figure 6 and can be compared to each facet superstructure of the TOh clusters shown in Figure 3.

The difference between $\mathrm{Dh}$ and $\mathrm{TOh}$ is the most pronounced around the composition of 0.66 where the $\mathrm{Dh}$ core is still disordered, as can be seen in Figure $4 \mathrm{c}$ in the bottom line and in Figure 5 as compared to Figure 1 looking at the core sublattices. Indeed, it seems that the $(\sqrt{3} \times \sqrt{3}) \mathrm{R} 30^{\circ}$ superstructure affects the core ordering more in the $\mathrm{Dh}$ than in the TOh structure because the (111) facets are more expanded in the TOh.

Icosahedra (Ih). Contrary to TOh and Dh shapes, Ih has only (111) facets and a core, which is much more distorted than $\mathrm{Dh}$; in place, one 5-fold axis and five tetrahedra, Ih has instead six 5-fold axis and 20 tetrahedra sharing the same corner, the central atom of the Ih. Different Ih compositions 
are displayed in Figure 7. If the core sublattice comparison with bulk sublattices is less significant because of the (a)

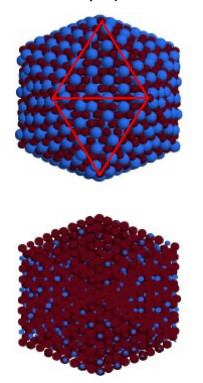

(b)

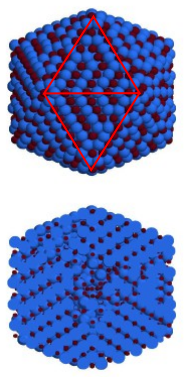

(C)

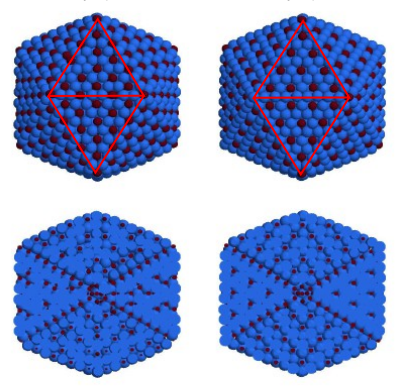

Figure 7. Snapshots of ordered structures $(\mathrm{a}-\mathrm{d})$ of $\mathrm{Ih}_{2869}$ at $300 \mathrm{~K}$ corresponding to the compositions pointed in Figure 8. The first row represents shell views, whereas the second row shows cutting views. The red triangles point to (111) facets. Co atoms are in red and Pt atoms in blue.

multitwinned structure of Ih, the surface sublattice occupations display interesting features, as shown in Figure 8. Even if the

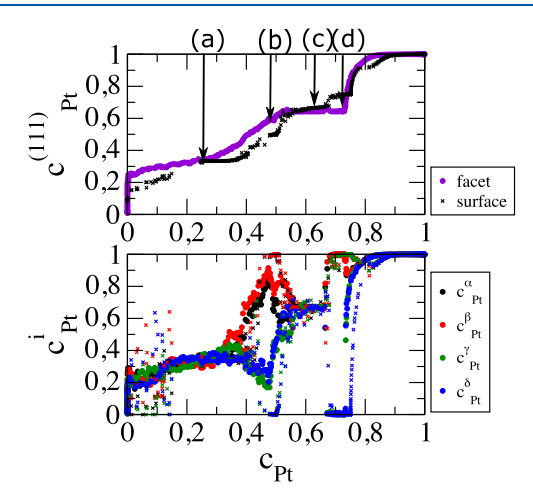

Figure 8. (111) facet $\mathrm{Pt}$ concentration of $\mathrm{Ih}_{2869}$ as compared to the one of the infinite (111) Co-Pt surface at $300 \mathrm{~K}$. Same graphs as Figure 3. Structures $(\mathrm{a}-\mathrm{d})$ are represented in Figure 7.

global $\mathrm{Pt}$ concentration at the Ih surface presents some discrepancies as compared to the one of the infinite (111) surface of $\mathrm{Co}-\mathrm{Pt}$ alloys, the sublattices are quite similar to those of (111) facets of $\mathrm{TOh}$ or Dh (Figures 3 and 6). In particular, we can observe the $(\sqrt{3} \times \sqrt{3}) \mathrm{R}_{30}^{\circ}$ twodimensional (2D) phase in Figure $7 \mathrm{a}, \mathrm{c}$, and the typical ordered $(111)$ surface structures as $\mathrm{p}(2 \times 1)$ (Figure $7 \mathrm{~b})$ and $\mathrm{p}(2 \times 2)$ (Figure $7 \mathrm{~d})$.

The surface ordering spreads in the core to give a certain core ordering, as can be seen in Figure $7 b$, with an alternance of pure planes along the [100] direction like the $\mathrm{L} 1_{0}$ ordered phase, and in Figure $7 \mathrm{~d}$, like the $\mathrm{L}_{2}$ ordered phase. The ordering starts from the surface and extends to the center of the core, where there is a local disorder due to the junction of different twinned tetrahedra.

The Ih nanoparticles display a localized ordering on each of the 20 tetrahedra, which is quite difficult to analyze both experimentally and theoretically. To our knowledge, there is no evidence of such ordering in the literature. From a theoretical point of view, multiply twinned morphologies of $\mathrm{FePt}$ and $\mathrm{CoPt}$ nanoparticles have been studied using large-scale density function theory calculations ${ }^{28}$ but the "ordered" icosahedron or the one with alternative concentric $\mathrm{Co} / \mathrm{Pt}$ shells are different from what we show here. Compared to another theoretical study on $\mathrm{Pd}-\mathrm{Au}$ nanoalloys ${ }^{36}$ where a similar core ordering is obtained on the TOh and Dh structures, the Ih ones do not reveal any core ordering and rather a $\mathrm{Pd}$ segregation in the center of the nanoparticle with a solid solution near the surface. This is a consequence of the strong chemical ordering tendency of the Co-Pt alloy as compared to the $\mathrm{Pd}-\mathrm{Au}$ one, which presents unexpected characteristics with ordering tendency but also a miscibility gap in the Pd-rich composition. $^{45}$

\section{CONCLUSIONS}

We presented a theoretical study of large-scale Co-Pt nanoalloys from 3 to $7 \mathrm{~nm}$ using a many-body interatomic potential and Monte Carlo simulations, sweeping the entire concentration at room temperature. By comparing the TOh, $\mathrm{Dh}$, and Ih nanoalloys to the bulk and to the surface alloys, we pointed out finite size effects and specific surface ordering.

On the Co-rich side of the TOh shape, the $\mathrm{L}_{2}$ phase in the core is disrupted. On the one side, the $(\sqrt{3} \times \sqrt{3}) \mathrm{R} 30^{\circ}$ structure on (111) facets is not compatible with the core ordering, on the other side, the $c(2 \times 2)$ structure on $(100)$ facets acts like a driving force to maintain the chemical ordering in the core. Therefore, a buffer layer appears between the surface and the ordered core, which tends to disappear by increasing cluster size. Furthermore, at the $\mathrm{CoPt}$ and $\mathrm{CoPt}_{3}$ stoichiometries, chemical ordering at the surface is the termination of the $\mathrm{Ll}_{0}$ ordered phase in the core. Indeed, $(100)$ and $(111)$ facets display, respectively, a $c(2 \times 2)$ and a $\mathrm{p}(2 \times 1)$ structure, compatible with the $\mathrm{L} 1_{0}$ order, except in the $c$-axis direction where the $L 1_{0}$ phase should lead to pure (100) facets. However, our model stabilizes the mixed composition on all of the (100) facets, pointing to the chemical frustration induced by the cluster morphology. Nevertheless, even if this represents a slight deviation from the pure $\mathrm{Ll}_{0}$ ordered phase, this is the best structure to get a magnetocrystalline anisotropy, improving magnetic properties for ultrahigh data storage, as related in experimental measurements. ${ }^{15,25}$ There is an experimental confirmation that we can get these $\mathrm{L} 1_{0}$ ordered clusters smaller than $4 \mathrm{~nm},{ }^{24}$ as the ones illustrated in Figures $2 \mathrm{~b}$ or $3 \mathrm{~b}$. For the $\mathrm{L}_{2}$ Pt-rich core, (100) facets have a $c(2 \times 2)$ structure or are Pt-pure, depending on the under or over $\mathrm{CoPt}_{3}$ stoichiometry, and (111) facets display a $\mathrm{p}(2 \times 2)$ phase. By increasing cluster size to 9879 atoms, facet behavior tends to the surface ones and the core to the bulk one.

In the case of 5-fold symmetry structures as decahedra, the behavior of (111) facets and of the core is similar to the ones of the truncated octahedra morphology because each twinned tetrahedron behaves like an fcc grain. This has been observed by high-resolution transmission electron microscopy, ${ }^{26}$ but, in this case, the magnetic performances are rather poor. Once again, by increasing cluster size, facet behavior tends to the surfaces ones and the core to the bulk one.

Icosahedra display a 5-fold symmetry structure, which multiplies the twinned tetrahedra from 5 to 20 . Nevertheless, at equiconcentration, we pointed out the $\mathrm{L}_{0}$ phase, multidomain, and locally disordered in the center of the core because of a geometrical frustration due to the junction of different twinned tetrahedra. On the surface, (111) facets are characterized by a $\mathrm{p}(2 \times 1)$ ordered structure with different orientations delimited by antiphase boundaries. On the Pt-rich 
side, the $\mathrm{L}_{2}$ phase is locally stabilized mainly due to the $\mathrm{p}(2 \times$ 2) (111) facet structure.

All of these structures with the $\mathrm{Pt}_{3}$ Co composition present a mixed surface composition, which has been shown to be highly active for the oxygen reduction reaction taking place at polymer electrolyte membrane fuel-cells cathodes. ${ }^{46}$ A sizedependence durability of the nanocatalysts where nanocatalysts with the intermediate size of around $8 \mathrm{~nm}$ present the best performance has also been reported. ${ }^{47}$ The structures are illustrated in Figure $1(6.8 \mathrm{~nm})$, Figure 2d, and Figures 3, 4, and $7 \mathrm{c}, \mathrm{d}$.

To conclude, we have shown that Co-Pt nanoalloys have all of the characteristics of chemically ordered structures with both core and surface ordering. However, the chemical ordering is not simply that observed in the bulk alloy. The surface and finite size effects lead to new 2D ordered superstructures like $(\sqrt{3} \times \sqrt{3}) \mathrm{R} 30^{\circ}$, which do not exist in the bulk alloys. It induces chemical disordering near facets, which is increasingly important for small sizes. Our Monte Carlo simulations evidenced core disordering due to surface ordering. In the 5-fold structures, twinned tetrahedra are locally ordered but the quasi-periodicity prevents a long-range ordered phase. This lack of long-range order is a clear disadvantage concerning magnetical properties where the $\mathrm{L} 1_{0}$ ordered phase is required to improve the magnetocrystalline anisotropy to reduce the superparamagnetism. On the other hand, catalytic properties depend on the local surface ordering, which is not altered by such finite size effects. As a result, Co$\mathrm{Pt}$ nanoalloys remain good candidates for electrocatalytic applications.

\section{AUTHOR INFORMATION}

\section{Corresponding Author}

Christine Mottet - Aix-Marseille Université, CNRS, CINaM UMR 7325, 13288 Marseille, France; 이이.org/00000002-7084-911X; Email: mottet@cinam.univ-mrs.fr

\section{Author \\ Alexis Front - Laboratoire d'Etude des Microstructures, ONERA-CNRS, UMR 104, University Paris-Saclay, 92322 Châtillon, France}

Complete contact information is available at: https://pubs.acs.org/10.1021/acs.jpcc.1c05701

\section{Notes}

The authors declare no competing financial interest.

\section{ACKNOWLEDGMENTS}

The authors warmly thank Bernard Legrand and Guy Tréglia for fruitful discussion and acknowledge support from the International Research Network-IRN "Nanoalloys" of CNRS. This work was granted access to the HPC resources of IDRIS under the allocation 2017-096829 made by GENCI. The authors acknowledge Tom Swinburne for a careful reading of the manuscript and improving its English.

\section{REFERENCES}

(1) Johnston, R.; Ferrando, R. Nanoalloys: From Theory to Application; RSC Publishing, 2008; Vol. 138.

(2) Ferrando, R.; Jellinek, J.; Johnston, R. Nanoalloys: from theory to applications of alloy clusters and nanoparticles. Chem. Rev. 2008, 108, 845-910.
(3) Alloyeau, D.; Mottet, C.; Ricolleau, C. Nanoalloys, Synthesis, Structure and Properties. In Engineering Materials; Springer-Verlag: London, 2012.

(4) Ferrando, R. Structure and Properties of Nanoalloys. In Frontiers of Nanoscience, Palmer, R., Ed.; Elsevier: London, 2016; Vol. 10.

(5) Petit, C.; Repain, V. Nanoalloys, Synthesis, Structure and Properties, Alloyeau, D.; Mottet, C.; Ricolleau, C., Eds.; SpringerVerlag: London, 2012; p 1.

(6) Xin, H. L.; Alayoglu, S.; Tao, R.; Genc, A.; Wang, C.-M.; Kovarik, L.; Stach, E. A.; Wang, L. W.; Salmeron, M.; Somorjai, G. A.; et al. Revealing the Atomic Restructuring of $\mathrm{Pt}-\mathrm{Co}$ Nanoparticles. Nano Lett. 2014, 14, 3203-3207.

(7) Chi, M.; Wang, C.; Lei, Y.; Wang, G.; Li, D.; More, K. L.; Lupini, A.; Allard, L. F.; Markovic, N. M.; Stamenkovic, V. R. Surface faceting and elemental diffusion behaviour at atomic scale for alloy nanoparticles during in situ annealing. Nat. Commun. 2015, 6, No. 8925.

(8) Pirart, J.; Front, A.; Rapetti, D.; Andreazza, P.; Mottet, C.; Ferrando, R.; Andreazza-Vignolle, C. Reversed size-dependent stabilization of ordered nanophases. Nat. Commun. 2019, 10, No. 1982.

(9) Penuelas, J.; Andreazza, P.; Andreazza-Vignolle, C.; Tolentino, H. C. N.; De Santis, M.; Mottet, C. Controlling Structure and Morphology of CoPt Nanoparticles through Dynamical or Static Coalescence Effects. Phys. Rev. Lett. 2008, 100, No. 115502.

(10) Andreazza, P.; Mottet, C.; Andreazza-Vignolle, C.; Penuelas, J.; Tolentino, H. C. N.; De Santis, M.; Felici, R.; Bouet, N. Probing nanoscale structural and order/disorder phase transitions of supported Co-Pt clusters under annealing. Phys. Rev. B 2010, 82, $155453-155460$.

(11) Andreazza, P.; Pierron-Bohnes, V.; Tournus, F.; AndreazzaVignolle, C.; Dupuis, V. Structure and order in cobalt/platinum-type nanoalloys: from thin films to supported clusters. Surf. Sci. Rep. 2015, $70,188-258$.

(12) Debe, M. Electrocatalyst approaches and challenges for automotive fuel cells. Nature 2012, 486, 43-51.

(13) Lebedeva, M.; Pierron-Bohnes, V.; Goyhenex, C.; Papaefthimiou, V.; Zafeiratos, S.; Nazmutdinov, R.; Costa, V. D.; Acosta, M.; Zosiak, L.; Kozubski, R.; et al. Effect of the chemical order on the electrocatalytic activity of model ptco electrodes in the oxygen reduction reaction. Electrochim. Acta 2013, 108, 605-616.

(14) Jamet, M.; Wernsdorfer, W.; Thirion, C.; Mailly, D.; Dupuis, V.; Mélinon, P.; Pérez, A. Magnetic anisotropy of a single cobalt nanocluster. Phys. Rev. Lett. 2001, 86, No. 4676.

(15) Tournus, F.; Tamion, A.; Blanc, N.; Hannour, A.; Bardotti, L.; Prével, B.; Ohresser, P.; Bonet, E.; Epicier, T.; Dupuis, V. Evidence of 110 chemical order in CoPt nanoclusters: Direct observation and magnetic signature. Phys. Rev. B 2008, 77, No. 144411.

(16) Tournus, F.; Blanc, N.; Tamion, A.; Hillenkamp, M.; Dupuis, V. Dispersion of magnetic anisotropy in size-selected CoPt clusters. Phys. Rev. B 2010, 81, No. 220405.

(17) Delalande, M.; Guinel, M. J.-F.; Allard, L. F.; Delattre, A.; Le Bris, R.; Samson, Y.; Bayle-Guillemaud, P.; Reiss, P. L10 Ordering of Ultrasmall Fept Nanoparticles Revealed by TEM In Situ Annealing. J. Phys. Chem. C 2012, 116, 6866-6872.

(18) Demortière, A.; Petit, C. First synthesis by liquid-liquid phase transfer of magnetic $\mathrm{Co}_{x} \mathrm{pt}_{1-x}$ nanoalloys. Langmuir 2007, 23, 85758584.

(19) Favre, L.; Dupuis, V.; Bernstein, E.; Mélinon, P.; Pérez, A.; Stanescu, S.; Epicier, T.; Simon, J.-P.; Babonneau, D.; Tonnerre, J.M.; et al. Structural and magnetic properties of CoPt mixed clusters. Phys. Rev. B 2006, 74, No. 014439.

(20) Rohart, S.; Raufast, C.; Favre, L.; Bernstein, E.; Bonet, E.; Dupuis, V. Magnetic anisotropy of $\mathrm{Co}_{x} \mathrm{Pt}_{1-x}$ clusters embedded in a matrix: Influences of the cluster chemical composition and the matrix nature. Phys. Rev. B 2006, 74, No. 104408.

(21) Tamion, A.; Hillenkamp, M.; Tournus, F.; Bonet, E.; Dupuis, V. Accurate determination of the magnetic anisotropy in clusterassembled nanostructures. Appl. Phys. Lett. 2009, 95, No. 062503. 
(22) Alloyeau, D.; Ricolleau, C.; Mottet, C.; Oikawa, T.; Langlois, C.; Le Bouar, Y.; Braidy, N.; Loiseau, A. Size and shape effects on the order-disorder phase transition in CoPt nanoparticles. Nat. Mater. 2009, 8, 940-947.

(23) Alloyeau, D.; Prévot, G.; Le Bouar, Y.; Oikawa, T.; Langlois, C.; Loiseau, A.; Ricolleau, C. Ostwald ripening in nanoalloys: When thermodynamics drives a size-dependent particle composition. Phys. Rev. Lett. 2010, 105, No. 255901.

(24) Blanc, N.; Tournus, F.; Dupuis, V.; Epicier, T. Measuring the L10 chemical order parameter of a single CoPt nanoparticle smaller than 4 nm. Phys. Rev. B 2011, 83, No. 092403.

(25) Bigot, J.-Y.; Kesserwan, H.; Haltié, V.; Ersen, O.; Moldovan, M.; Kim, T.; Jang, J.-T.; Cheon, J. Magnetic Properties of Annealed Core-Shell CoPt Nanoparticles. Nano Lett. 2012, 12, 1189-1197.

(26) Tournus, F.; Sato, K.; Epicier, T.; Kono, T.; Dupuis, V. Multi$\mathrm{L1}_{0}$ domain CoPt and fept nanoparticles revealed by electron microscopy. Phys. Rev. Lett. 2013, 110, No. 055501.

(27) Blanc, N.; Diaz-Sánchez, L. E.; Ramos, A. Y.; Tournus, F.; Tolentino, H. C. N.; De Santis, M.; Proux, O.; Tamion, A.; TuaillonCombes, J.; Bardotti, L.; et al. Element-specific quantitative determination of the local atomic order in CoPt alloy nanoparticles: Experiment and theory. Phys. Rev. B 2013, 87, No. 155412.

(28) Gruner, M. E.; Rollmann, G.; Entel, P.; Farle, M. Multiply twinned morphologies of FePt and CoPt nanoparticles. Phys. Rev. Lett. 2008, 100, No. 087203.

(29) Barcaro, G.; Ferrando, R.; Fortunelli, A.; Rossi, G. Exotic supported CoPt nanostructures: From clusters to wires. J. Phys. Chem. Lett. 2010, 1, 111-115.

(30) Montejano-Carrizales, J.; Aguilera-Granja, F.; Goyhenex, C.; Pierron-Bohnes, V.; Moran-Lopez, J. Structural, electronic and magnetic properties of $\mathrm{Co}_{n} \mathrm{Pt}_{M-n}$, for $\mathrm{M}=13,19$, and 55, from first principles. J. Magn. Magn. Mater. 2014, 355, 215-224.

(31) Calvo, F.; Mottet, C. Order-disorder transition in Co-Pt nanoparticles: Coexistence, transition states, and finite-size effects. Phys. Rev. B 2011, 84, No. 035409.

(32) Lopes, A.; Tréglia, G.; Mottet, C.; Legrand, B. Ordering and surface segregation in $\mathrm{Co}_{1-c} \mathrm{Pt}_{c}$ nanoparticles: A theoretical study from surface alloys to nanoalloys. Phys. Rev. B 2015, 91, No. 035407.

(33) Chepulskii, R. V.; Butler, W. H. Tuning of L10 atomic order in Co-Pt nanoparticles: Ab initio insights. Phys. Rev. B 2012, 86, No. 155401.

(34) Kovács, G.; Kozlov, S. M.; Matolinova, I.; Vorokhta, M.; Matolin, V.; Neyman, K. M. Revealing chemical ordering in $\mathrm{Pt}-\mathrm{Co}$ nanoparticles using electronic structure calculations and X-ray photoelectron spectroscopy. Phys. Chem. Chem. Phys. 2015, 17, 28298-28311.

(35) Front, A.; Legrand, B.; Tréglia, G.; Mottet, C. Bidimensional phases in Co-Pt surface alloys: A theoretical study of ordering and surface segregation. Surf. Sci. 2019, 679, 128-138.

(36) Zhu, B.; Guesmi, H.; Creuze, J.; Legrand, B.; Mottet, C. Crossover among structural motifs in Pd-Au nanoalloys. Phys. Chem. Chem. Phys. 2015, 17, 28129-28136.

(37) Rosato, V.; Guillopé, M.; Legrand, B. Thermodynamical and structural properties of f.c.c. transition metals using a simple tightbinding model. Philos. Mag. A 1989, 59, 321-336.

(38) Goyhenex, C.; Bulou, H.; Deville, J.-P.; Tréglia, G. Pt/ $\mathrm{Co}(0001)$ superstructures in the submonolayer range: A tight-binding quenched-molecular-dynamics study. Phys. Rev. B 1999, 60, 27812788.

(39) Bochicchio, D.; Ferrando, R. Size-dependent transition to highsymmetry chiral structures in $\mathrm{AgCu}, \mathrm{AgCo}, \mathrm{AgNi}$, and $\mathrm{AuNi}$ nanoalloys. Nano Lett. 2010, 10, 4211-4216.

(40) Bochicchio, D.; Ferrando, R. Morphological instability of coreshell metallic nanoparticles. Phys. Rev. B 2013, 87, No. 165435.

(41) Gauthier, Y.; Baudoing-Savois, R.; Bugnard, J.; Hebenstreit, W.; Schmid, M.; Varga, P. Surf. Sci. 2000, 466, 155.

(42) Bugnard, J.; Gauthier, Y.; Baudoing-Savois, R. Segregation profile and surface structure of Pt35Co65(110): Pt25Co75(110) revisited. Surf. Sci. 1995, 344, 42-50.
(43) Hultgren, R.; Desai, P. D.; Hawkins, D. T.; Gleiser, M.; Kelley, K. K. Selected Values of the Thermodynamic Properties of Binary Alloys, American Society for Metals, Berkeley; Jossey-Bass Publishers, 1981; p 777.

(44) Leroux, C.; Loiseau, A.; Broddin, A.; Tendeloo, G. V. Electron microscopy study of the coherent two-phase mixtures L10 + L12, in Co-Pt alloys. Philos. Mag. B 1991, 64, 57-82.

(45) Berthier, F.; Creuze, J.; Gabard, T.; Legrand, B.; Marinica, M.C.; Mottet, C. Order-disorder or phase-separation transition: Analysis of the Au-Pd system by the effective site energy model. Phys. Rev. B 2019, 99, No. 014108.

(46) Wang, C.; van der Vliet, D.; Chang, K.-C.; You, H.; Strmcnik, D.; Schlueter, J.; Markovic, N.; Stamenkovic, V. Monodisperse Pt3co Nanoparticles as a Catalyst for the Oxygen Reduction Reaction: SizeDependent Activity. J. Phys. Chem. C 2009, 113, 19365-19368.

(47) Gummalla, M.; Ball, S. C.; Condit, D. A.; Rasouli, S.; Yu, K.; Ferreira, P.; Myers, D.; Yang, Z. Effect of Particle Size and Operating Conditions on Pt3Co Pemfc Cathode Catalyst Durability. Catalysts 2015, 5, 926-948. 